\title{
AcsA-AcsB: The Core of the Cellulose Synthase Complex from Gluconacetobacter hansenii ATCC23769
}

\author{
John B. McManus ${ }^{\mathrm{a}}$, Ying Deng ${ }^{\mathrm{a}}$, Nivedita Nagachar ${ }^{\mathrm{a}}$, Teh-hui Kao ${ }^{\mathrm{a}}$, and Ming Tien ${ }^{\mathrm{a}, \mathrm{b}}$
}

${ }^{a}$ Department of Biochemistry and Molecular Biology, 305 South Frear, University Park, The Pennsylvania State University, PA 16802, USA.

${ }^{\mathrm{b}}$ Corresponding author: 305 South Frear, University Park, The Pennsylvania State University, PA 16802, USA. Tel: + 1814863 1165; E-mail: mxt3@ @su.edu; Fax: + 18148637024.

(C) 2015. This manuscript version is made available under the Elsevier user license http://www.elsevier.com/open-access/userlicense/1.0/ 


\begin{abstract}
The Gram-negative bacterium, Gluconacetobacter hansenii, produces cellulose of exceptionally high crystallinity in comparison to the cellulose of higher plants. This bacterial cellulose is synthesized and extruded into the extracellular medium by the cellulose synthase complex (CSC). The catalytic component of this complex is encoded by the gene AcsAB. However, several other genes are known to encode proteins critical to cellulose synthesis and are likely components of the bacterial CSC. We have purified an active heterodimer AcsA-AcsB from $G$. hansenii ATCC23769 to homogeneity by two different methods. With the purified protein, we have determined how it is post-translationally processed, forming the active heterodimer AcsAAcsB. Additionally, we have performed steady-state kinetic studies on the AcsA-AcsB complex. Finally through mutagenesis studies, we have explored the roles of the postulated CSC proteins AcsC, AcsD, and CcpAx.
\end{abstract}

Keywords: Cellulose; Gluconacetobacter hansenii; Cell Wall; acetobacter; glycosyltransferase Abbreviations: CSC - Cellulose Synthase Complex; BC - Bacterial Cellulose; AcsA-AcsB the active $G$. hansenii cellulose synthase heterodimer 


\section{INTRODUCTION}

Cellulose is a polymer of $\beta-1,4$-linked glucose units. It is the most abundant renewable polymer on Earth [1], being the major component of the plant cell wall [2]. Research effort into cellulose synthesis has intensified in recent years, mainly due to interest in the conversion of cellulose into bioethanol $[3,4]$. Cellulose is not only synthesized by higher plants; microbes also synthesize cellulose [5, 6]. This microbial process has served as a model for cellulose synthesis in the plant system. In both systems, cellulose is synthesized by membrane-bound glycosyltransferases, utilizing UDP-glucose to form the glucan polymer [7]. The individual cellulose chains can then coalesce to form crystalline cellulose. The crystallinity is due to inter- and intra-molecular hydrogen bonding, such that water cannot penetrate the cellulose chains [8]. In plants, the crystallinity of cellulose imparts tensile strength and rigidity to the cell wall [9].

Knowledge on bacterial cellulose synthesis has predominantly come from research with two bacteria. Recent work with bacterial cellulose synthesis provided the first crystal structure of a cellulose synthase, that of Rhodobacter sphaeroides [10]. However, the most work has been performed with the bacterium Gluconacetobacter hansenii which synthesizes crystalline cellulose [11], similar to that found in plants but with a higher degree of crystallinity [12, 13]. Genetic and biochemical studies have shown that G. hansenii synthesizes cellulose through a multi-protein cellulose synthase complex (CSC), as is the case in higher plants. Electron microscopic studies have shown that putative CSCs form a membrane-associated linear array [5, 14]. Our recent sequencing of the complete genome has enhanced the use of this bacterium as a model system [15]. 
Mutagenesis, biochemical, and structural studies have shown that, for the G. hansenii CSC, the active site is contained within the AcsA protein and that this protein is associated with AcsB [10, 16, 17]. In addition to Acs $A$ and $A c s B$, mutagenesis studies have shown that $A \operatorname{cs} C, A c s D$, CcpAx, and CmcAx are essential protein components for cellulose synthesis in G. hansenii [14, 18, 19]. AcsC is thought to be a porin-like protein allowing for extrusion of the cellulose chain through the outer membrane [18]. AcsD is periplasm-localized, and forms a homooctamer, with a central pore large enough to hold four glucan chains [20]. The small protein, $\mathrm{CcpAx}$, has been shown to associate with AcsD, and may play a role in the formation of the linear array of CSCs [14]. The fourth protein implicated in the production of cellulose is the endo-glucanase $\mathrm{CmcAx}$, whose disruption leads to a reduction in cellulose synthesis and production of twisted cellulose ribbons [21].

In this study, we have purified the active cellulose synthase AcsA and AcsB from G. hansenii ATCC23769 to homogeneity by two different methods. With the purified proteins, we have determined the post-translational cleavage site of the AcsAB peptide by $\mathrm{N}$-terminal sequencing of AcsB and we have performed steady state kinetic analysis of this enzyme. To our knowledge, this is the first study in which AcsA and AcsB of G. hansenii ATCC23769 have been homologously expressed and affinity-purified. Finally, our results show that AcsC, AcsD, and CcpAx, while not impacting catalytic activity, most likely impact the secretion of cellulose and stability of the CSC.

\section{MATERIALS AND METHODS}

Cultures. G. hansenii cells expressing the dodecahistidine-tagged $\mathrm{AcsAB}$ were grown at $30^{\circ} \mathrm{C}$ in a $60 \mathrm{~L}$ fermenter in Schraam-Hestrin (SH) medium [22] containing $20 \mathrm{mg} / \mathrm{L}$ of tetracycline to an 
absorbance of 1.5 at $600 \mathrm{~nm}$, and the cells were harvested by centrifugation. All other cultures were grown in $\mathrm{SH}$ medium with antibiotics for the appropriate selectable marker at $30^{\circ} \mathrm{C}$ with shaking at $250 \mathrm{rpm}$ to an absorbance of $0.6-0.8$ at $600 \mathrm{~nm}$, and the cells were harvested.

Cloning, Heterologous Expression, and Purification of AcsD. The gene encoding AcsD was amplified from G. hansenii genomic DNA by PCR using primers for AcsD (Table S1). The 0.5 $\mathrm{kb}$ fragment was ligated into a NdeI/XhoI digested modified pET21a vector, which added Cterminal hexahistidine tag preceded by a thrombin cleavage sequence to the AcsD peptide, and expressed in BL21(DE3) cells. The protein was purified by Ni-NTA chromatography and quantified using a molar extinction coefficient of $26,470 \mathrm{~cm}^{-1} \mathrm{M}^{-1}$ at $280 \mathrm{~nm}$.

Cloning and Purification of cсpAx. The fragment chosen for cloning was based on the active peptide identified by the work of Sunagawa et al. [14]. The ccpAx gene was obtained by PCR (Table S1). The $186 \mathrm{bp}$ fragment was cloned into the modified pET21a vector also with a hexahistidine tag at the C-terminus. Expression and purification methods were identical to those used for AcsD.

Homologous Expression of AcsA-AcsB-his tag. A 3' flanking fragment of AcsAB was amplified using primers (Table S1) to G. hansenii ATCC23769 genomic DNA. The tetC gene was amplified using primers (Table S1) to the pUCD2 plasmid. The two PCR fragments and BamHI/EcoRI digested pUC18 were ligated using the In-Fusion HD Cloning Kit (Clontech), and the resulting construct was introduced into wild-type G. hansenii ATCC23769 cells via electroporation. The transformants were selected on SH/tetracycline agar plates. A schematic diagram of this procedure is available in supplemental materials (Fig. S1). 
AcsD Insertional Mutant via Double Homologous Recombination. Two flanking fragments of $A c s D$ were amplified using primer pairs (Table S1) to the genomic DNA of wild-type $G$. hansenii ATCC23769. The tetC gene was amplified as described above. The three PCR fragments and BamHI/EcoRI digested pUC18 were ligated using the In-Fusion HD Cloning Kit, and the resulting construct was introduced into wild-type G. hansenii ATCC23769 cells via electroporation. The transformants were selected on SH/tetracycline agar plates. Cloning and sequencing of the PCR fragments were performed as described by Deng et al. [19]. Protein Concentration Determination. The protein concentrations of purified AcsA-AcsB and AcsD were determined by their molar extinction coefficient as described elsewhere in the paper. For all other samples, the protein concentrations were determined by a modified method of Lowry et al. [23] using bovine serum albumin as a standard. In brief, $80 \mu \mathrm{L}$ of working solution (20 parts $5 \%$ sodium carbonate, 10 parts $10 \%$ SDS, 8 parts 1 M sodium hydroxide, 1 part $2 \%$ sodium potassium tartrate, and 1 part $1 \%$ cupric sulfate pentahydrate) was mixed with $80 \mu \mathrm{L}$ of sample, and developed with $40 \mu \mathrm{L}$ Folin phenol reagent (diluted 1:10). Samples were analyzed using a Spectramax plate reader.

AcsA-AcsB Purification by Product Entrapment. Product entrapment, first described by Kang et al. [24], was performed as follows. All steps were performed at $4^{\circ} \mathrm{C}$, unless otherwise noted. Harvested cells ( $1 \mathrm{~g}$ wet weight) were resuspended in $10 \mathrm{~mL}$ of PMC buffer $(20 \mathrm{mM}$ sodium phosphate, $\mathrm{pH} 7.5,5 \mathrm{mM} \mathrm{MgCl} 2,5 \mathrm{mM}$ cellobiose), and lysed in a microfluidizer two times at 20,000 psi. Total lysate was clarified by centrifugation at 2,800 $\times \mathrm{g}$ for $30 \mathrm{~min}$. Total membrane was isolated by centrifugation at $250,000 \times \mathrm{g}$ for $1.5 \mathrm{~h}$ and then resuspended $(1 \mathrm{~g})$ in $5 \mathrm{~mL}$ solubilization buffer (20 mM sodium phosphate, $\mathrm{pH} 7.5,5 \mathrm{mM} \mathrm{MgCl}_{2}, 5 \mathrm{mM}$ cellobiose, $10 \%$ glycerol, $2 \%$ Triton) using a glass homogenizer. After $1 \mathrm{~h}$ of gentle rotary shaking, the total 
membrane was subject to centrifugation at $100,000 \times \mathrm{g}$ for $1 \mathrm{~h}$. The supernatant $(6.8 \mathrm{~mL})$ was then mixed with $3.4 \mathrm{~mL}$ of $60 \mu \mathrm{M}$ cyclic-di-GMP and $0.2 \mathrm{~mL}$ of $100 \mathrm{mM}$ UDP-glucose. The entire mixture was layered on top of $2.6 \mathrm{~mL}$ of PMC buffer containing $30 \%$ glycerol. The mixture was incubated at $30^{\circ} \mathrm{C}$ for $11 \mathrm{~min}$ and then incubated on ice for $2 \mathrm{~h}$. The mixture was centrifuged in a swing bucket rotor at $50,000 \times \mathrm{g}$ for $20 \mathrm{~min}$ and the entire supernatant and glycerol cushion were removed. The pellet was resuspended in $1.0 \mathrm{~mL}$ of resuspension buffer (20 mM phosphate, $\mathrm{pH} 7.5,5 \mathrm{mM} \mathrm{MgCl} 2,5 \mathrm{mM}$ cellobiose, $10 \%$ glycerol, $0.1 \%$ dodecylmaltoside) and centrifuged at $16,000 \times \mathrm{g}$ for $10 \mathrm{~min}$ at $4^{\circ} \mathrm{C}$. The pellet was again resuspended in $0.5 \mathrm{~mL}$ resuspension buffer and stored at $-80^{\circ} \mathrm{C}$.

AcsA-AcsB Purification by Affinity Chromatography. All steps were performed at $4^{\circ} \mathrm{C}$ unless otherwise noted. Total membrane was collected and solubilized in solubilization buffer as described above for product entrapment. The AcsA-AcsB protein was purified from solubilized membrane $(20 \mathrm{~mL})$ with TALON cobalt affinity resin (Clontech) $(1 \mathrm{~mL})$ using $20 \mathrm{mM}$ sodium phosphate, $\mathrm{pH}$ 7.5, $5 \mathrm{mM} \mathrm{MgCl} 2,5 \mathrm{mM}$ cellobiose, $10 \%$ glycerol, $100 \mathrm{mM} \mathrm{NaCl}, 20 \mathrm{mM}$ imidazole, $0.1 \%$ dodecylmaltoside as the wash buffer (total volume $15 \mathrm{~mL}$ ). AcsA-AcsB was eluted from the column with the same buffer, except with a gradient of 20-120 mM imidazole (total volume of $10 \mathrm{~mL}$ ). Purity was determined by SDS-PAGE and Coomassie blue staining. The sample was then concentrated with a $50 \mathrm{kDa}$ cut-off centrifugal concentrator and stored at $80^{\circ} \mathrm{C}$. The protein concentration was determined by absorbance at $280 \mathrm{~nm}$ using a molar extinction coefficient of $195,745 \mathrm{~cm}^{-1} \mathrm{M}^{-1}$.

Preparation of Total Membrane. Total membrane that was used for enzyme assays was prepared as described by Myers et al. [25] and stored in $10 \mathrm{mM}$ Tris-Cl, $\mathrm{pH} 8.0$ with 20\% glycerol at - 
$80^{\circ} \mathrm{C}$. Note that this method is different than the large scale preparations for enzyme purification as described above.

Measurement of Cellulose Synthase Activity by ${ }^{14} \mathrm{C}$-Glucose Incorporation. Unless otherwise noted, activity was measured at $30^{\circ} \mathrm{C}$ in $40 \mathrm{mM}$ Tris- $\mathrm{Cl}$, $\mathrm{pH} 8.0,15 \mathrm{mM} \mathrm{MgCl}_{2}, 1 \mathrm{mM} \mathrm{CaCl}_{2}, 12$ $\mu \mathrm{M}$ cyclic-di-GMP, and $5 \mathrm{mM}$ UDP-glucose in a total volume of $300 \mu \mathrm{L}$. The stock UDP- ${ }^{14} \mathrm{C}-$ glucose was diluted with non-radioactive UDP-glucose to a specific activity of $3.7 \times 10^{6}$ $\mathrm{Bq} / \mathrm{mmol}$. Reactions were terminated at $10 \mathrm{~min}$ by the addition of $2 \mathrm{~mL}$ of $1 \mathrm{M}$ sodium hydroxide. Next, $20 \mathrm{mg}$ of cellulose was added, and the mix was boiled for $20 \mathrm{~min}$. After cooling to $\mathrm{RT}, 4 \mathrm{~mL}$ of $0.5 \mathrm{M} \mathrm{HCl}$ was added and the entire mixture was filtered through a glass filter $(1.2 \mu \mathrm{m}$ pore size). The filter was washed with $30 \mathrm{~mL}$ water, $10 \mathrm{~mL}$ methanol, allowed to dry, and was added to $5 \mathrm{~mL}$ ScintiVerse (Fisher Scientific) scintillation fluid. Radioactivity was quantified with a Beckman Coulter LS6500 liquid scintillation counter.

Cellulose Synthase Activity as Determined by Enzyme-Coupled Assay. Unless otherwise noted, activity was measured at $30^{\circ} \mathrm{C}$ in $40 \mathrm{mM}$ Tris-Cl, $\mathrm{pH} 8.0,15 \mathrm{mM} \mathrm{MgCl}_{2}, 1 \mathrm{mM} \mathrm{CaCl}_{2}, 12 \mu \mathrm{M}$ cyclic-di-GMP, $2 \mathrm{mM}$ phosphoenolpyruvate, $0.5 \mathrm{mM} \mathrm{NADH}$, with $20 \mathrm{U}$ of pyruvate kinase and 20 U lactate dehydrogenase (Sigma-Aldrich), and 5 mM UDP-glucose in a total volume of 200 $\mu \mathrm{L}$. The assay mix was pre-incubated at $30^{\circ} \mathrm{C}$ for $10 \mathrm{~min}$ and the reaction was initiated by the addition of AcsA-AcsB ( $0.25 \mu \mathrm{M}$ final concentration). Each turnover results in release of UDP from UDP-glucose. The UDP is converted to UTP by pyruvate kinase and the pyruvate is then reduced by the NADH-utilizing enzyme lactate dehydrogenase. Enzyme activity is measured by the decrease in absorbance at $340 \mathrm{~nm}$ associated with NADH oxidation [26]. 
SDS-PAGE and Western Blot Analysis. Protein samples (20-40 $\mu \mathrm{g}$ per lane or 1-4 $\mu \mathrm{g}$ per lane for pure AcsA-AcsB) were incubated with lithium dodecyl sulfate (LDS) loading buffer and incubated on ice for $1 \mathrm{~h}$, or with sodium dodecyl sulfate (SDS) loading buffer and boiled for 10 min. Samples were loaded onto a $12 \%$ polyacrylamide gel and run at $300 \mathrm{~V}$. For Western blot analysis, the proteins were transferred to nitrocellulose at $100 \mathrm{~V}, 0.5 \mathrm{~A}$ for $1 \mathrm{~h}$ and immunestained with the indicated primary antibodies followed by a secondary alkaline phosphataseconjugated anti-rabbit IgG (Sigma Aldrich) incubation. The blots were developed with ECF reagent (GE Healthcare) for 0.5-2 min and visualized on a fluorescence typhoon scanner.

Statistical Analysis. To determine statistical significance in the activity and protein levels reported in Fig. 6 and 7, a pairwise comparison between the means of the wild type control and each mutant was performed using Welch's $t$-test (two tailed, unequal variance).

Miscellaneous Methods. N-terminal sequencing of AcsB was performed by Edman degradation at the Iowa State University Protein Facility. For cell lysis using trichloroacetic acid-acetone and total protein analysis, G. hansenii ATCC23769 cells were grown to an absorbance of 0.8 at 600 $\mathrm{nm}$, collected by centrifugation, and washed in $50 \mathrm{mM}$ phosphate buffer, $\mathrm{pH}$ 7.5. Approximately $35 \mathrm{mg}$ of cells were lysed in $1 \mathrm{~mL}$ of $10 \%$ trichloroacetic acid in acetone. Total protein was collected by centrifugation and washed three times in acetone. The pellet was resuspended in LDS loading buffer, and Western blot analysis was performed as previously described.

\section{RESULTS}

Purification of AcsA-AcsB by Product Entrapment. The method of product entrapment had been previously used for purification of both cellulose synthase [16] and callose synthase [27]. First, 
the total membrane fraction from G. hansenii ATCC23769 was isolated by ultracentrifugation [24] and solubilized in 2\% Triton X-100. Cellulose synthesis was initiated upon addition of the substrate, UDP-glucose, and the activator, cyclic-di-GMP. The enzyme, cellulose synthase, was entrapped with the newly-synthesized insoluble cellulose, which then could be isolated by centrifugation.

The entrapped proteins were subjected to SDS-PAGE and the proteins visualized by Coomassie blue staining (Fig. 1). A previous report indicated that the AcsA-AcsB protein is unstable to boiling in SDS [28]. As such, we compared preparation of the entrapped protein with SDS or LDS. Boiling with $1 \%$ SDS, in contrast to incubating on ice with $1 \%$ LDS prior to electrophoresis on SDS-PAGE, yielded only one detectable protein band (Fig. 1A). A second protein band was detected when the pellet was incubated on ice in $1 \%$ LDS (Fig. 1A). To confirm that these two protein bands are products of the $A \operatorname{cs} A B$ gene, we performed Western blot analysis using anti-AcsA $\mathrm{A}_{1}$ (raised against residues 129-397 of AcsAB), anti-AcsA 3 (raised against a synthetic peptide of residues 581-600 of AcsAB), and anti-AcsB (raised against residues 610-1550 of AcsAB) (Fig.1B). The preparation and specificity of these antibodies had been documented in our previous paper [29]. The Western blot revealed an 85 kDa crossreactive band when using anti-Acs $\mathrm{A}_{1}$ and anti-Acs $\mathrm{A}_{3}$. These bands corresponded to AcsA, which contains the active site. The $100 \mathrm{kDa}$ band was cross-reactive with the anti-AcsB antibody and was identified as AcsB. Neither the Coomassie stain nor Western blot revealed a $169 \mathrm{kDa}$ band, corresponding to the unprocessed AcsA-AcsB, suggesting that processing must have occurred quickly after translation. In order to confirm that cleavage is not an artifact of total membrane isolation and solubilization, we lysed whole cells directly in $10 \%$ trichloroacetic acid- 
acetone. The total protein, along with purified AcsA-AcsB, was subject to Western blot analysis using anti-AcsB (Fig. S2). The Western blot revealed only a $100 \mathrm{kDa}$ band corresponding to the processed AcsB peptide.

Post-translational Cleavage Site. The cleavage site for AcsA-AcsB was determined by Nterminal sequencing via Edman degradation of the gel-purified AcsB. The first five amino acids identified were Ala-Ser-Ala-Pro-Arg (Fig. 2). Therefore, AcsA-AcsB cleavage occurs between residues 757 and 758. Fig. 2 shows that the AQA cleavage site is also conserved in the other cellulose synthase-containing bacteria.

His-tag Purification of AcsA-AcsB. The AcsA-AcsB cellulose synthase isolated by product entrapment was greater than $95 \%$ pure based on Coomassie blue staining of samples separated by SDS-PAGE. However, use of this enzyme preparation for kinetic analysis required removal of the cellulose product associated with this enzyme. This proved to be problematic as incubation with cellulases resulted in substantial loss of enzyme activity. Consequently, we transformed G. hansenii [19] with an AcsAB-containing construct and through homologous recombination, generated a C-terminally dodecahistine-tagged AcsB. This resulted in disruption of the downstream $\operatorname{Acs} C$ gene via insertion of a tetracycline resistance marker. Total membrane was isolated from this transformant, solubilized, and subjected to affinity chromatography with a TALON cobalt column. The total activity increased upon solubilization of the total membrane with detergent. This finding is consistent with work by Hashimoto et al. [30], showing that cellulose synthesis activity increases upon solubilization of total membrane to varying degrees associated with detergent type. Fractions from each step of the purification were assayed for 
cellulose synthase activity by incorporation of ${ }^{14} \mathrm{C}$-glucose into alkaline-insoluble product [26]. Only a small fraction of the total activity was recovered from the total lysate (Table 1). The purified AcsA and AcsB were subjected to SDS-PAGE after treatment with LDS (instead of SDS). The Coomassie blue-stained gel revealed two bands that accounted for over $90 \%$ of the total band intensity (Fig. 3A). These two bands were confirmed by Western blot to be AcsA and AcsB (Fig. 3B). Furthermore, both product entrapment and affinity purification show no bands of similar intensity to AcsA and AcsB or corresponding to the expected molecular weights of ccpax, AcsD, or AcsC (Fig. S3).

Kinetic Analysis of AcsA-AcsB. With purified cellulose synthase preparations, the enzyme activity can be monitored in a continuous assay with coupled enzymes, as described in Materials and Methods.

The $\underline{K}_{d}$ for cyclic-di-GMP was determined by varying the concentration of this activator, at saturating UDP-glucose concentration ( $5 \mathrm{mM})$, and measuring the activity. As shown in Fig. 4B, the $\underline{K}_{d_{-}}$was calculated to be $0.18 \pm 0.01 \mu \mathrm{M}$. Enzyme activity was measured at varying concentrations of UDP-glucose (Fig. 4A) in the presence of saturating levels of the activator molecule, cyclic-di-GMP $(12 \mu \mathrm{M}) . \mathrm{A} \underline{K_{M}}$ of $0.28 \pm 0.07 \mathrm{mM}$ was calculated for UDP-glucose and a $\underline{k}_{\text {cat_- }}$ of $1.72 \pm 0.06 \mathrm{~s}^{-1}$. These values yield a $k_{\text {cat }} / K_{M}$ (second order rate constant) of $6,100 \pm$ $1,600 \mathrm{M}^{-1} \mathrm{~s}^{-1}$. The numerical values for both Michaelis-Menten curves are provided in Table S2. The reproducibility of the $\underline{k}_{\text {cat }}$ value among different preparations was tested with three other purified enzyme preparations. The mean and standard deviation for four preparations under saturating substrate conditions was $1.60 \pm 0.50 \mathrm{~s}^{-1}$. 
Activation Energy for Cellulose Synthase. For the measurement of the activation energy, enzyme activity was measured by ${ }^{14} \mathrm{C}$-glucose incorporation [26]. This circumvented possible complications from temperature changes on the downstream enzymes (pyruvate kinase etc.). The reaction velocity, under saturating UDP-glucose concentration, was measured over the temperature range 15 to $30^{\circ} \mathrm{C}$ at $\mathrm{pH} 8.0$. The Arrhenius plot was linear in this temperature range and yielded an activation energy of $76.5 \mathrm{~kJ} / \mathrm{mol}$ (Fig. 5).

Effect of AcsD, AcsC, and ccpAx on Enzyme Activity. Our results show that the purified AcsAAcsB proteins alone are sufficient to catalyze cellulose synthesis. However, genome sequencing has shown that $A \operatorname{cs} A B$ is part of an operon that includes $A \operatorname{cs} C$ [18]. Also, mutations in $A c s D$ and ccpAx greatly decrease the amount of cellulose synthesized in vivo $[14,18,19]$. As such, we further explored the effects of AcsC, AcsD, and CcpAx on AcsA-AcsB activity with both in vitro (purified protein) experiments and in vivo (mutagenesis) experiments. Mutations were generated by $\operatorname{Tn} 5$ transposon random mutagenesis as previously described [19]. These insertional mutations in $A c s C, A c s D$, and $\operatorname{ccp} A x$ resulted in significantly lowered, if not totally eliminated, cellulose production in whole cells in the medium $[18,19]$. The cellulose synthase activity of isolated membranes, containing both the cytoplasmic and outer membrane, was then measured by ${ }^{14} \mathrm{C}$-glucose incorporation in the mutant lines. For the AcsC mutant, the in vitro activity was not statistically different, when compared to that of wild type, with the AcsD showing a statistically reduced activity $(p$-value $<0.05)$ (Fig. 6). The in vitro synthase activity for the $\operatorname{ccpAx}$ knockout was significantly lowered ( $p$-value $<0.01$ ), showing only $33 \pm 4 \%$ of wild type (Fig. 6). We then examined the levels of AcsA and AcsB in these three mutants by 
Western blot analysis (Fig. 7A and 7B). The AcsA and AcsB levels in the total membrane fraction were similar to that in wild type for the $A \operatorname{cs} C$ and $A c s D$ mutants (Fig. 7C). Interestingly, the ccpAx mutant had no impact on the AcsA levels, but significantly decreased the AcsB levels relative to wild type ( $\underline{p \text {-value }}<0.01)$. We also tested the AcsA mutant which showed $20 \pm 4 \%$ the activity of wild type. When compared to wild type and each other mutant tested here, the

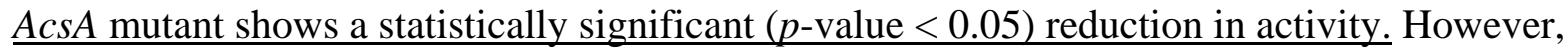
Western blot analysis revealed no band present for AcsA (Fig. 8).

To further assess the impact of AcsD and CcpAx on cellulose synthase activity, both proteins were expressed heterologously in E. coli and purified by Ni-affinity chromatography (Fig. 9A and 9B). Both proteins were added separately or together at a final concentration of $1 \mu \mathrm{M}$ to assay mixtures containing purified AcsA-AcsB. The rate of cellulose synthesis was assessed by the enzyme coupled assays monitoring NADH oxidation. In all cases, there was no apparent effect on activity (Table 2).

\section{DISCUSSION}

The Catalytic Core of the CSC. The isolation of AcsA-AcsB by product entrapment and by histag affinity methods are both analogous to a pull-down experiment. The only protein that AcsBhis pulled down was AcsA. Other proteins found to be essential for cellulose synthesis in vivo, AcsC, AcsD, and Ccpax were not pulled down with the AcsA-AcsB in our experiments. This does not preclude these proteins from being part of the CSC. However, if they are part of the CSC, their association must be relatively weak. Therefore, our results suggest that the minimal 
components needed for catalytic activity are AcsA and AcsB. This conclusion is in accord with results obtained for $R$. sphaeroides $\mathrm{Bcs} \mathrm{A}-\mathrm{BcsB}[31]$.

We were also able to heterologously express and purify AcsD and CcpAx. With the purified proteins, it was relatively easy to determine their impact on in vitro cellulose synthesis activity. Addition of these two proteins, either alone, or in combination, to the AcsA-AcsB cellulose synthesis reaction mixture, had no effect on the rate of cellulose synthesis. There again, this negative result does not preclude them from being part of the CSC.

Evidence for the involvement of these proteins comes from the effects of disruption of their genes on cellulose synthesis in vivo $[14,18,19]$. As has been previously postulated, AcsC is likely the outer membrane porin that allows for the extrusion of cellulose into the extracellular space [18]. Our results show that, while no in vivo cellulose can be detected in the AcsC mutant [19], the in vitro activity of the total membrane is similar to that of wild type. Also, the levels of both AcsA and AcsB appear to be unaffected, suggesting that AcsC has no effect on cellulose synthesis at the level of the glucan chain, nor does it affect the stability of AcsA-AcsB.

The other protein we examined for impact on cellulose synthesis activity was AcsD, which is a periplasmic localized protein [32]. The crystal structure of AcsD shows an octamer forming a doughnut shaped structure with four channels in the center leaving room for four glucan chains [20]. Previous studies have shown that in vivo cellulose production is reduced in mutants lacking AcsD [18], and it has been proposed that AcsD may play a role in the crystallization of the glucan chains [18]. Our results are consistent with these previous studies, showing decreased in 
vivo cellulose synthesis in the mutant (data not shown). However, the in vitro rate of cellulose synthesis catalyzed by the total membrane fraction from the mutant, while lower than that of wild type, was not eliminated. This reduction in activity is not apparently observed with Western blots showing similar levels of AcsA and AcsB. These results suggest that AcsD may have an impact on the rate at which AcsA-AcsB catalyzes the addition of glucose to the glucan chain. However, when purified AcsD is added to purified AcsA-AcsB, no increase in the rate of catalysis is observed. More importantly, we do not see any AcsD in either affinity-purified AcsA-AcsB or product-entrapped AcsA-AcsB. Therefore, while AcsD may play a role in the production of cellulose in vivo, it is not necessary for the synthesis of cellulose by AcsA-AcsB.

The CcpAx mutant has also been shown not to produce cellulose in vivo [14, 19, 21]. The work of Sunagawa et al. [14] has also suggested an association between CcpAx and AcsD where this association may play a key role in the formation of the linear array of the CSC. Our in vitro activity assay shows a reduction in activity when compared with wild type, as well as the AcsC and $A c s D$ mutants ( $p$-value $<0.01$ ). The background activity seen in the AcsA mutant is likely due to a second copy of $A \operatorname{cs} A B$ found in G. hansenii. We hypothesize that CcpAx, supported by the work of Sunagawa et al. [14], is critical for maintaining the linear cellulose synthase array and that in the absence of CcpAx, the array disperses and results in a decrease in levels of AcsB. The apparent reduction of AcsB protein (in the CcpAx mutant), relative to wild type, cannot be due to lower expression levels of AcsB relative to AcsA. This is because these two proteins are translated as a single peptide. The more likely explanation is instability (rapid turnover) of AcsB in the absence of $\mathrm{CcpAx}$ by mechanisms yet unknown. Our work, as well as work done by 
Omadjela et al. [31] on BcsA-BcsB, suggests that, while the precise function of AcsB is unclear, it is critical for cellulose synthesis.

Processing of AcsA-AcsB. Previous work by Lin et al. [28], using product entrapment on solubilized membrane from G. hansenii ATCC53582, demonstrated that AcsA-AcsB is posttranslationally modified to yield AcsA and AcsB of molecular masses $83 \mathrm{kDa}$ and $93 \mathrm{kDa}$, respectively. Using similar entrapment methods to isolated AcsA-AcsB, our work here with $G$. hansenii ATCC23769, showed that cleavage occurs between residues 757 and 758 . These residues are on the carboxyl side of an AQA amino acid triplet. Examination of $B \operatorname{cs} B$ genes from closely-related bacteria reveal that they encode a conserved AQA triplets which are preceded by periplasmic targeting sequences [33]. AxA triplets are common signal peptidase

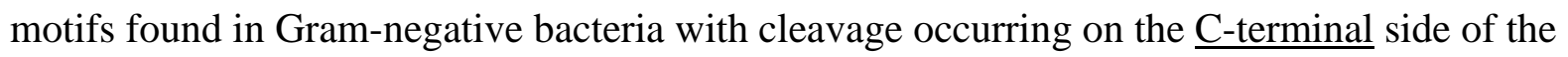
amino acid triplet [34]. Cleavage after an AxA motif suggests that a signal peptidase, found on the periplasmic side of the cytoplasmic membrane, is responsible for processing. The present results for AcsA are in contrast to our previous work showing that AcsA is further processed into two smaller fragments of $46 \mathrm{kDa}$ and $34 \mathrm{kDa}$ [29]. The weakness and variability of these two smaller bands on Western blots was the result of AcsA being unstable to boiling in the presence of SDS. When LDS was used as a denaturant, as per Lin and Brown [28] under lower temperature conditions, we were then able to detect the $83 \mathrm{kDa}$ band associated with AcsA.

The cleavage site determined in this study occurs upstream of the site identified for ATCC53582 by Saxena et al. [35]. The difference in cleavage site may be due to the fact that we used a different strain ATCC23769 in this work. However, another possible explanation is that when 
these workers isolated the membrane fraction, they employed a step with trypsin treatment prior to entrapment [35]. This is consistent with their finding that the cleavage site is located at a lysine residue [35].

Kinetic Analysis of AcsA-AcsB. The AcsA-AcsB his-tagged protein was used for all kinetic analysis. The mean and standard deviation of the $\underline{k}_{c a t}$ value for three preparations was $1.60 \pm$ $0.50 \mathrm{~s}^{-1}$. Our value is lower than two other published $\underline{k}_{c a t}$ values for cellulose synthases. Even though these two reports are of the same source, $R$. sphaeroides, their values are drastically different. A value of $90 \mathrm{~s}^{-1}$ has been reported [31] and more recently, another group reported a $k_{c a t}$ an order of magnitude lower [36]. If not due to experimental error, there are many possible reasons for the large differences in the $k_{c a t}$ values. One study determined the kinetic parameters in detergent micelles [36] whereas the other were in nanodiscs [31]. Our $k_{\text {cat }}$ value of $1.6 \mathrm{~s}^{-1}$ for the G. hansenii enzyme is again another order of magnitude lower. Due to the large variation in the $k_{\text {cat }}$ values for the $R$. spaeroides enzyme, it is difficult to assess whether indeed the $G$. hansenii enzyme is much slower. However, it is worthwhile mentioning that the $R$. sphaeroides enzyme purifies as a monomer and the G. hansenii is known to form linear arrays in vivo [5]. This may impact the crystallinity of the resultant cellulose [13] and as such, may be relevant because cellulose crystallization has been proposed to be the rate limiting step in cellulose synthesis [37]. However, providing a mechanistic rationale for the different $k_{\text {cat }}$ values at this time would be premature on our part.

The $\underline{K}_{\underline{M}}$ value for UDP-glucose, determined in this report, is in agreement with the values, previously determined for purified $R$. sphaeroides $\mathrm{B} \operatorname{cs} \mathrm{A}-\mathrm{B} \operatorname{csB}[31]$ and for the total membrane 
fraction from G. hansenii ATCC53582 [38], of $0.5 \mathrm{mM}$ and $0.21 \mathrm{mM}$, respectively. These values are consistent with physiological concentrations of 1-2 mM for UDP-glucose [39]. The $\underline{K}_{\underline{d}}$ value determined here for cyclic-di-GMP is in the micromolar region and lower, consistent with the value of $1.0 \mu \mathrm{M}$, determined for $R$. sphaeroides $\mathrm{B} \operatorname{csA} \mathrm{A} \operatorname{BcsB}$ [31], also consistent with physiological concentrations of 0.2-2.5 $\mu \mathrm{M}$ for cyclic-di-GMP [40] in Gram-negative bacteria. Interestingly, an earlier report shows cyclic-di-GMP involved in an allosteric interaction [41]. In contrast, our work here, as well as the report by Omadjela et al. [31], show a Michaelis-Menten type interaction with cyclic-di-GMP. The earlier study by Ross et al. [41] was performed using total membrane instead of the purified protein used in this study. This may be a possible explanation for the different results obtained in the two studies. However, our experiments here do not allow us rationalize the two results. Furthermore, the mode of action of cyclic-di-GMP is not a focus of our study.

The $76.5 \mathrm{~kJ} / \mathrm{mol}$ activation energy we determined is similar to those calculated for other UDPsugar inverting glycosyltransferases operating through $\mathrm{S}_{\mathrm{N}} 2$ single displacement mechanisms [42].

In conclusion, our work confirms that AcsA-AcsB is the catalytic core of the CSC in $G$. hansenii. Aside from CcpAx, mutations in AcsC and AcsD do not eliminate the ability of total membranes to catalyze cellulose synthesis. The role of CcpAx, in accord with the work of Sunagawa et al. [14], is likely to be involved in proper maintenance of the linear array of the CSCs. In its absence, we observe the apparent instability of the AcsB protein. We have also shown that the nascent AcsAB peptide is post-translationally processed into the active AcsA- 
$\underline{\text { AcsB heterodimer and that both peptides are necessary and sufficient (alone) for } \beta \text {-1,4-glucan }}$

synthesis.

\section{ACKNOWLEDGEMENTS}

This material is based upon work supported as part of The Center for LignoCellulose Structure and Formation, an Energy Frontier Research Center funded by the U.S. Department of Energy, Office of Science, Office of Basic Energy Sciences under Award Number DE-SC0001090. The Penn State Shared Fermentation Facility is acknowledged for large scale production of enzymes.

\section{REFERENCES CITED}

[1] Aristidou A, Penttila M. Metabolic engineering applications to renewable resource utilization. Curr Opin Biotech. 2000;11:187-98.

[2] Somerville C. Cellulose synthesis in higher plants. Annu Rev Cell Dev Bi. 2006;22:53-78.

[3] Youngs H, Somerville C. Development of feedstocks for cellulosic biofuels. F1000 biology reports. 2012;4:10.

[4] Youngs H, Somerville C. Plant science. Best practices for biofuels. Science. 2014;344:10956.

[5] Brown RM, Montezinos D. Cellulose microfibrils: visualization of biosynthetic and orienting complexes in association with the plasma membrane. Proc Natl Acad Sci USA. 1976;73:143-7.

[6] Brown RM, Willison JHM, Richardson CL. Cellulose biosynthesis in Acetobacter xylinum: visualization of the site of synthesis and direct measurement of the in vivo process. Proc Natl Acad Sci USA. 1976;73:4565-9.

[7] Carpita NC. Update on Mechanisms of Plant Cell Wall Biosynthesis: How Plants Make Cellulose and Other (1 -> 4)-beta-D-Glycans. Plant Physiol. 2011;155:171-84.

[8] Mwaikambo LY, Ansell MP. The determination of porosity and cellulose content of plant fibers by density methods. J Mater Sci Lett. 2001;20:2095-6.

[9] Cosgrove DJ. Growth of the plant cell wall. Nat Rev Mol Cell Bio. 2005;6:850-61.

[10] Morgan JL, Strumillo J, Zimmer J. Crystallographic snapshot of cellulose synthesis and membrane translocation. Nature. 2013;493:181-6.

[11] Saxena IM, Brown RM, Jr. Cellulose biosynthesis: current views and evolving concepts. Annals of botany. 2005;96:9-21.

[12] Harris D, DeBolt S. Relative Crystallinity of Plant Biomass: Studies on Assembly, Adaptation and Acclimation. Plos One. 2008;3.

[13] Park S, Johnson DK, Davis MF. Cellulose crystallinity index measured by solid state C-13 nuclear magnetic resonance and X-ray diffraction. Abstr Pap Am Chem S. 2010;239. 
[14] Sunagawa N, Fujiwara T, Yoda T, Kawano S, Satoh Y, Yao M, et al. Cellulose complementing factor (Ccp) is a new member of the cellulose synthase complex (terminal complex) in Acetobacter xylinum. J Biosci Bioeng. 2013;115:607-12.

[15] Iyer PR, Geib SM, Catchmark J, Kao TH, Tien M. Genome sequence of a celluloseproducing bacterium, Gluconacetobacter hansenii ATCC 23769. Journal of bacteriology. 2010;192:4256-7.

[16] Lin FC, Brown RM, Drake RR, Haley BE. Identification of the uridine 5'-diphosphoglucose (UDP-Glc) binding subunit of cellulose synthase in Acetobacter xylinum using the photoaffinity probe 5-azido-UDP-Glc. J Biol Chem. 1990;265:4782-4.

[17] Saxena IM, Lin FC, Brown RM, Jr. Cloning and sequencing of the cellulose synthase catalytic subunit gene of Acetobacter xylinum. Plant molecular biology. 1990;15:673-83.

[18] Saxena IM, Kudlicka K, Okuda K, Brown RM. Characterization of genes in the cellulosesynthesizing operon (acs operon) of Acetobacter xylinum: implications for cellulose crystallization. Journal of bacteriology. 1994;176:5735-52.

[19] Deng Y, Nagachar N, Xiao CW, Tien M, Kao TH. Identification and Characterization of Non-Cellulose-Producing Mutants of Gluconacetobacter hansenii Generated by Tn5 Transposon Mutagenesis. Journal of bacteriology. 2013;195:5072-83.

[20] Hu SQ, Gao YG, Tajima K, Sunagawa N, Zhou Y, Kawano S, et al. Structure of bacterial cellulose synthase subunit D octamer with four inner passageways. Proc Natl Acad Sci USA. 2010;107:17957-61.

[21] Nakai T, Sugano Y, Shoda M, Sakakibara H, Oiwa K, Tuzi S, et al. Formation of Highly Twisted Ribbons in a Carboxymethylcellulase Gene-Disrupted Strain of a Cellulose-Producing Bacterium. J Bactiol. 2013;195:958-64.

[22] Schramm M, Hestrin S. Synthesis of cellulose by Acetobacter xylinum. 1. Micromethod for the determination of celluloses. The Biochemical journal. 1954;56:163-6.

[23] Lowry OH, Rosebrough NJ, Farr AL, Randall RJ. Protein Measurement with the Folin Phenol Reagent. J Biol Chem. 1951;193:265-75.

[24] Kang MS, Elango N, Mattia E, Auyoung J, Robbins PW, Cabib E. Isolation of chitin synthetase from Saccharomyces cerevisiae. Purification of an enzyme by entrapment in the reaction product. J Biol Chem. 1984;259:4966-72.

[25] Myers CR, Myers JM. Localization of cytochromes to the outer membrane of anaerobically grown Shewanella putrefaciens MR-1. Journal of bacteriology. 1992;174:3429-38.

[26] Brown C, Leijon F, Bulone V. Radiometric and spectrophotometric in vitro assays of glycosyltransferases involved in plant cell wall carbohydrate biosynthesis. Nat Protoc.

2012;7:1634-50.

[27] Turner A, Bacic A, Harris PJ, Read SM. Membrane fractionation and enrichment of callose synthase from pollen tubes of Nicotiana alata Link et Otto. Planta. 1998;205:380-8.

[28] Lin FC, Brown RM. Purification of cellulose synthase from Acetobacter xylinum. In:

Schuerch C, editor. Cellulose and Wood-Chemistry and Technology. New York: John Wiley and Sons, Inc.; 1989. p. 473-92.

[29] Iyer PR, Liu YA, Deng Y, McManus JB, Kao TH, Tien M. Processing of cellulose synthase (AcsAB) from Gluconacetobacter hansenii 23769. Arch Biochem Biophys. 2013;529:92-8.

[30] Hashimoto A, Shimono K, Horikawa Y, Ichikawa T, Wada M, Imai T, et al. Extraction of cellulose-synthesizing activity of Gluconacetobacter xylinus by alkylmaltoside. Carbohydr Res. 2011;346:2760-8. 
[31] Omadjela O, Narahari A, Strumillo J, Melida H, Mazur O, Bulone V, et al. BcsA and BcsB form the catalytically active core of bacterial cellulose synthase sufficient for in vitro cellulose synthesis. Proceedings of the National Academy of Sciences of the United States of America. 2013;110:17856-61.

[32] Iyer PR, Catchmark J, Brown NR, Tien M. Biochemical localization of a protein involved in synthesis of Gluconacetobacter hansenii cellulose. Cellulose. 2011;18:739-47.

[33] Martoglio B, Dobberstein B. Signal sequences: more than just greasy peptides. Trends Cell Biol. 1998;8:410-5.

[34] Tuteja R. Type I signal peptidase: An overview. Arch Biochem Biophys. 2005;441:107-11. [35] Saxena IM, Lin FC, Brown RM. Identification of a new gene in an operon for cellulose biosynthesis in Acetobacter xylinum. Plant molecular biology. 1991;16:947-54.

[36] Erbakan M, Curtis BS, Tracy Nixon B, Kumar M, Curtis WR. Advancing Rhodobacter sphaeroides as a platform for expression of functional membrane proteins. Protein expression and purification. 2015;15:127-8.

[37] Benziman M, Haigler CH, Brown RM, White AR, Cooper KM. Cellulose biogenesis: Polymerization and crystallization are coupled processes in Acetobacter xylinum. Proceedings of the National Academy of Sciences of the United States of America. 1980;77:6678-82.

[38] Bureau TE, Brown RM. In vitro synthesis of cellulose II from a cytoplasmic membrane fraction of Acetobacter xylinum. Proceedings of the National Academy of Sciences of the United States of America. 1987;84:6985-9.

[39] Buckstein MH, He J, Rubin H. Characterization of nucleotide pools as a function of physiological state in Escherichia coli. Journal of bacteriology. 2008;190:718-26.

[40] Koestler BJ, Waters CM. Bile acids and bicarbonate inversely regulate intracellular cyclic di-GMP in Vibrio cholerae. Infect Immun. 2014;82:3002-14.

[41] Ross P, Aloni Y, Weinhouse C, Michaeli D, Weinbergerohana P, Meyer R, et al. An unusual guanyl oligonucleotide regulates cellulose synthesis in Acetobacter xylinum. Febs Lett. 1985;186:191-6.

[42] Lairson LL, Henrissat B, Davies GJ, Withers SG. Glycosyltransferases: Structures, functions, and mechanisms. Annu Rev Biochem. 2008;77:521-55. 
Table 1. Purification table for AcsA-AcsB.*

\begin{tabular}{|c|c|c|c|c|c|c|}
\hline Sample & $\begin{array}{c}\text { Volume } \\
(\mathrm{ml})\end{array}$ & $\begin{array}{c}\text { Protein } \\
(\mathrm{mg} / \mathrm{ml})\end{array}$ & $\begin{array}{c}\text { Specific } \\
\text { Activity } \\
(\mathrm{nmol} / \mathrm{min} / \mathrm{mg})\end{array}$ & $\begin{array}{c}\text { Total Activity } \\
(\mathrm{nmol} / \mathrm{min})\end{array}$ & $\begin{array}{c}\text { Fold } \\
\text { Purification }\end{array}$ & $\begin{array}{c}\text { Percent } \\
\text { Recovered }\end{array}$ \\
\hline Lysate & 600 & 14.78 & 3.76 & 33803 & 1 & 100 \\
\hline $\begin{array}{c}\text { total } \\
\text { membrane }\end{array}$ & 120 & 27.40 & 8.17 & 14707 & 2.2 & 44 \\
\hline $\begin{array}{c}\text { total } \\
\text { membrane 1h } \\
\text { solubilization }\end{array}$ & 200 & 16.46 & 9.21 & 27645 & 2.5 & 82 \\
\hline Solubilized & 175 & 11.48 & 8.49 & 22292 & 2.3 & 66 \\
\hline Co-TALLON & 1.2 & 1.04 & 656.71 & 1182 & 175 & 3 \\
\hline
\end{tabular}

*Cellulose synthesis was measured by ${ }^{14} \mathrm{C}$-glucose incorporation as described in Materials and Methods.

"After solubilization in detergent, the total membrane was then centrifuged as described in Materials and Method and the supernatant was assayed for cellulose synthase activity.

Table 2. Effect of different proteins on cellulose synthase activity of AcsA-AcsB.*

\begin{tabular}{|l|l|}
\hline Additions & Velocity, $\mathrm{s}^{-1}$ \\
\hline None & $1.69 \pm 0.09$ \\
\hline AcsD & $1.67 \pm 0.04$ \\
\hline CcpAx & $1.61 \pm 0.04$ \\
\hline AcsD+CcpAx & $1.78 \pm 0.01$ \\
\hline
\end{tabular}

*Cellulose synthase activity was measured by monitoring NADH oxidation as described in Materials and Methods. AcsD and CcpAx, when added, were at $1 \mu \mathrm{M}$ final concentration.

\section{FIGURE LEGENDS}

Fig. 1. Isolation of AcsA-AcsB by product entrapment. Panel A: Coomassie-stained SDSpolyacrylamide gel showing total membrane (lane 1), soluble total membrane (lane 2), and entrapped product after either boiling in SDS (lane 3) or incubating on ice in LDS (lane 4). Panel B: Entrapped product was incubated on ice in LDS and then subjected to Western blot analysis with anti-AcsA 1 (lane 5), anti-AcsA 3 (lane 6), or anti-AcsB (lane 7). 
Fig. 2. Sequence alignment between AcsA-AcsB and three homologs from other cellulosesynthesizing bacteria. The cleavage site, AQA, is shown in bold (cleavage occurs after AQA). Note that all the other three sequences start with methionine, encoded by their respective initiation codons. Thus, these AcsB homologs are encoded by separate genes from their respective $A \operatorname{cs} A$ genes, and they are not post-translationally processed.

Fig. 3. Purification of AcsA-AcsB by cobalt affinity chromatography. The total membrane was isolated as described in Materials and Methods, and AcsA-AcsB was purified by cobalt affinity chromatography. Panel A shows Coomassie-blue stained SDS-polyacrylamide gels of the total membrane (lane 1), solubilized total membrane (lane 2), and purified AcsA-AcsB (lane 3). Panel

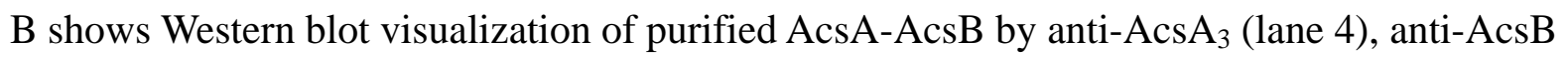
(lane 5), or anti-his (lane 6).

Fig. 4. Steady-state kinetic analysis of AcsA-AcsB. Panel A: Effect of UDP-glucose concentration on enzyme velocity. Panel B: Effect of cyclic-di-GMP concentration on enzyme velocity. Reaction rates were measured in enzyme-coupled assays by monitoring decrease in absorbance at $340 \mathrm{~nm}$ associated with NADH oxidation as described in Materials and Methods. Rates are expressed as velocity $\left(\mathrm{s}^{-1}\right)$. Data shown is from mean and standard deviation of three separate assays.

Fig. 5. Arrhenius plot. Enzyme velocities were measured and reported as velocity $\left(\mathrm{s}^{-1}\right)$. The plot is of $\ln$ (velocity) versus the inverse temperature. Reaction rates were measured at various 
temperatures by monitoring ${ }^{14} \mathrm{C}$-glucose incorporation into cellulose from UDP-glucose as described in Materials and Methods.

Fig. 6. Quantification of cellulose synthase activity in wild type (WT) and insertional mutants. The total membrane from each of the mutants (as designated in the figure with a $\Delta$ before the name of the gene) and from wild type were isolated. Cellulose synthase activity was measured by ${ }^{14} \mathrm{C}$-glucose incorporation as described in Materials and Methods and expressed as nmol glucose incorporated per minute per milligram protein $(\mathrm{nmol} / \mathrm{min} / \mathrm{mg})$. Each assay contained a total of $120 \mu \mathrm{g}$ of total membrane. Results show mean and standard deviation from three biological replicates (mean value for wild type was $25.0 \pm 2.6 \mathrm{nmol} / \mathrm{min} / \mathrm{mg}$ ). $\underline{* \text { Statistically }}$ significant difference in activity when compared to WT using Welch's T-test, $p$-value $<0.05$. **Statistically significant difference in activity when compared to WT using Welch's T-test, $p$ value $<0.01$.

Fig. 7. Western blot visualization and quantification of SDS-polyacrylamide gels of total membrane from wild type, AcsC, AcsD, or $c c p A x$ insertional mutants. A total $30 \mu \mathrm{g}$ of total membrane protein was loaded in each lane. Panel A: Western blot visualized with anti-AcsA, of total membrane from wild type (lane1), AcsC mutant (lane 2), AcsD mutant (lane 3), and CcpAx mutant (lane 4). Panel B: Western blot visualized with anti-AcsB, of total membrane from wild type (lane 6), AcsC mutant (lane 7), AcsD mutant (lane 8), and $c c p A x$ mutant (lane 9). The three blots in each panel are of three biological replicates. Molecular mass markers and purified AcsA-AcsB are indicated with labels in lanes 5 and 10. Panel C: The band intensities of blots shown in Panel A and B were quantified using Image $\mathrm{J}$ and plotted as a percent of wild type 
for AcsA (black) and AcsB (grey). The insertional mutants are as labeled with a $\Delta$.

**Statistically significant difference in protein levels when compared to WT using Welch's T-

test, $p$-value $<0.01$.

Fig. 8. Western blot analysis of insertional mutant of AcsA. Panel A was visualized with anti-Acs $\mathrm{A}_{3}$, of a blot containing pure AcsA-AcsB (lane1), wild-type total membrane (lane 2), and AcsA mutant total membrane (lane 3). Results show no AcsA band present for the AcsA mutant as indicated by the arrow. Panel B was visualized with anti-AcsB, of a blot containing pure AcsA-AcsB (lane 4), wild-type total membrane (lane 5), and AcsA mutant total membrane (lane 6). Results show no AcsB band present for the AcsA mutant as indicated by the arrow.

Fig. 9. Coomassie blue stained SDS-polyacrylamide gels of purified (Panel A) AcsD and (Panel B) CcpAx. AcsD and CcpAx were expressed and purified as described in Materials and Methods. The purified protein was then subject to SDS-PAGE analysis. 


\section{FIGURES}

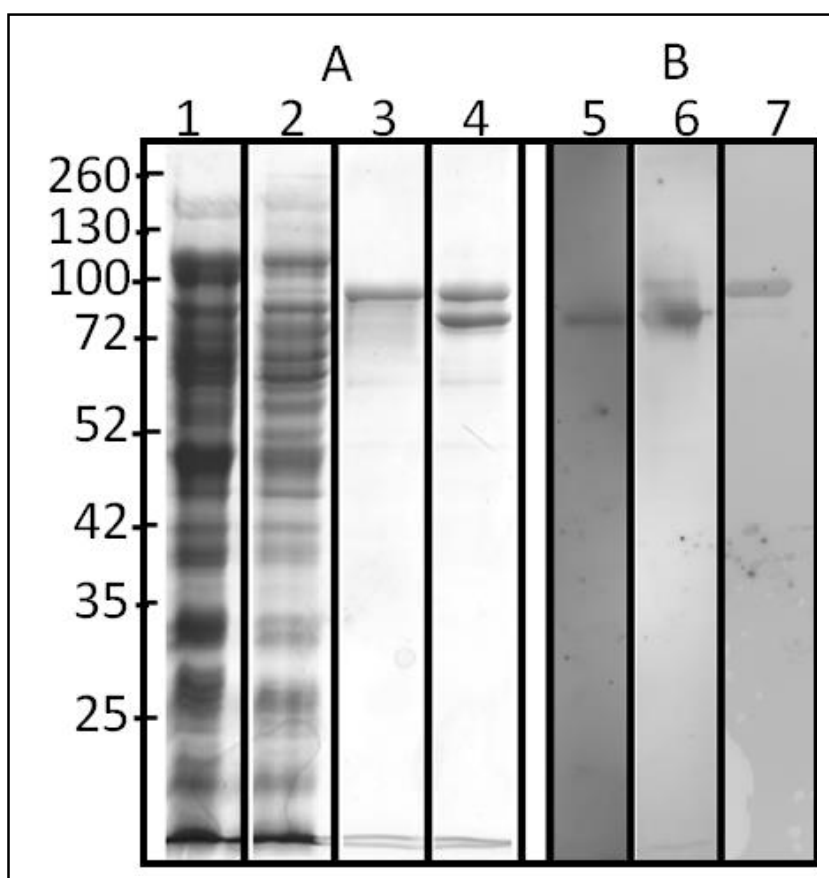

Fig. 1.

AcsAB
G. xylinus
G. oboediens
G. europaeu

$\begin{array}{rrr}731 & \text { RKERVLKGTV } & \text { KMVSLLALLT } \\ 1 & \mathrm{M} & \text { KMVSLIALLV } \\ 1 & \mathrm{M} & \text { KMVSLIALLV } \\ 1 & \text { MRPRDM } & \text { KMVSLIALLV }\end{array}$

SSAOAASA PRAVAA 1036

FATGAQAAP. ...IAS 24

FATGAQAAP. ..IIAS 24

. europaeu

Fig. 2 


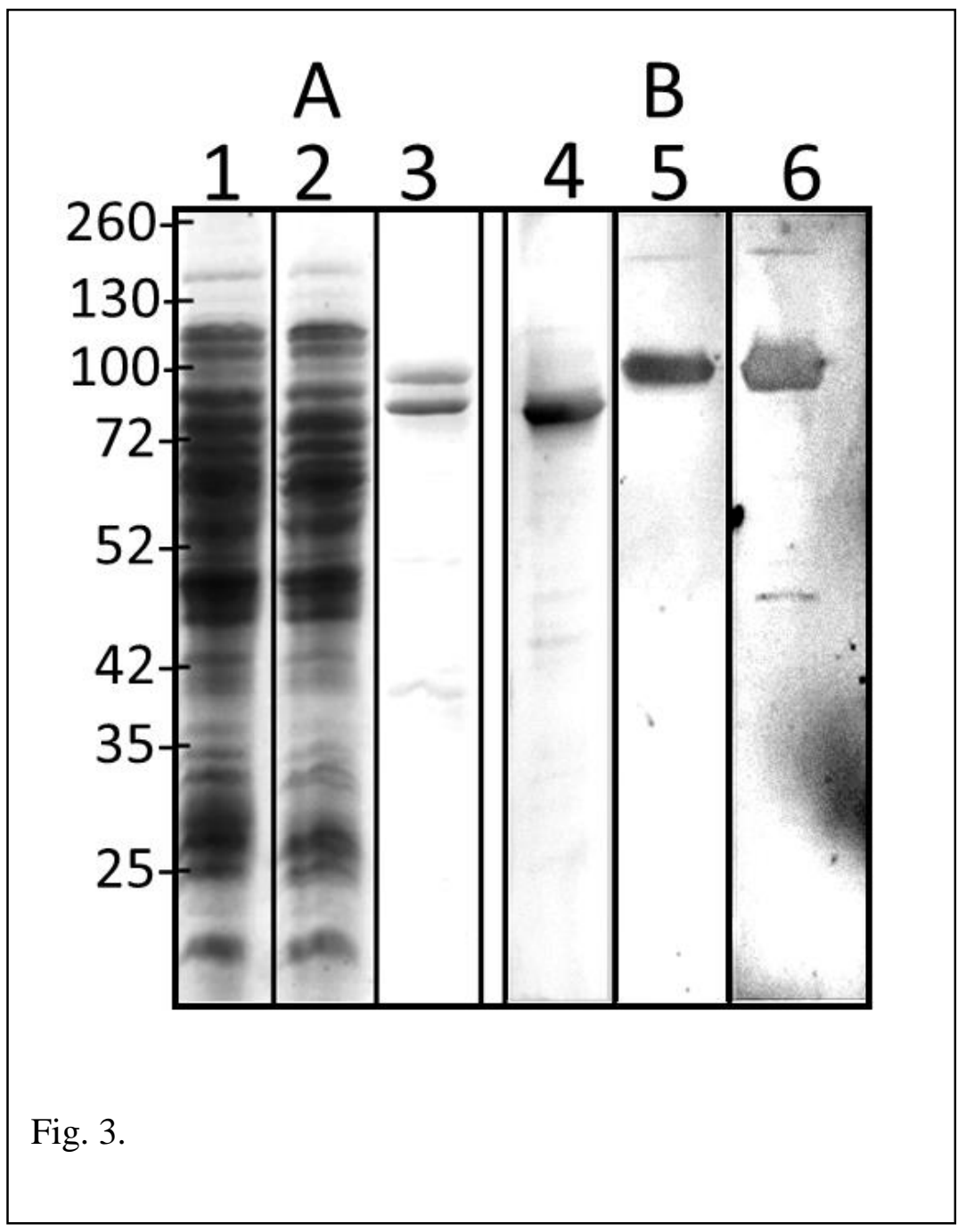




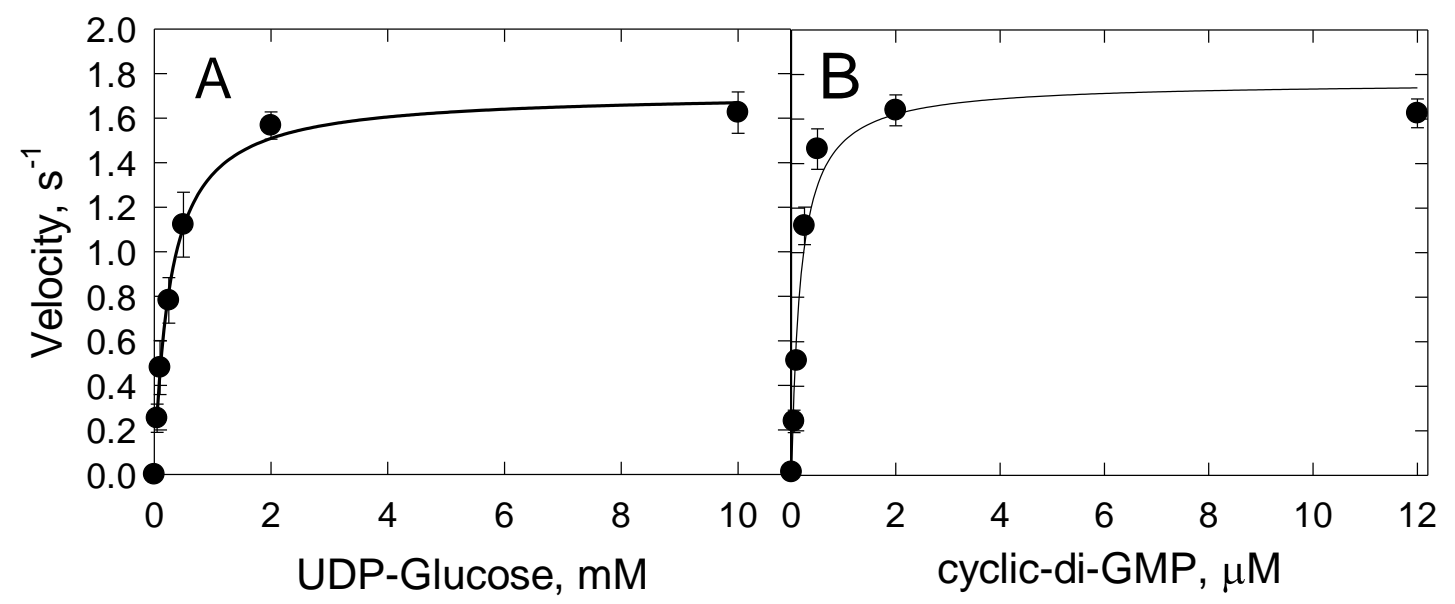

Fig. 4.

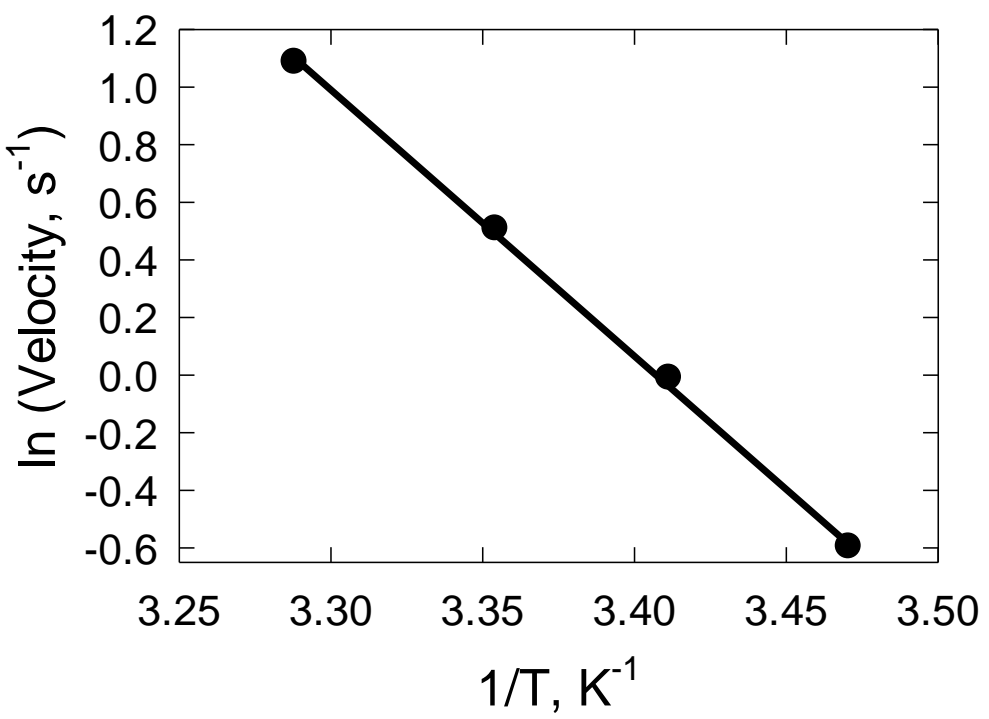

Fig. 5. 

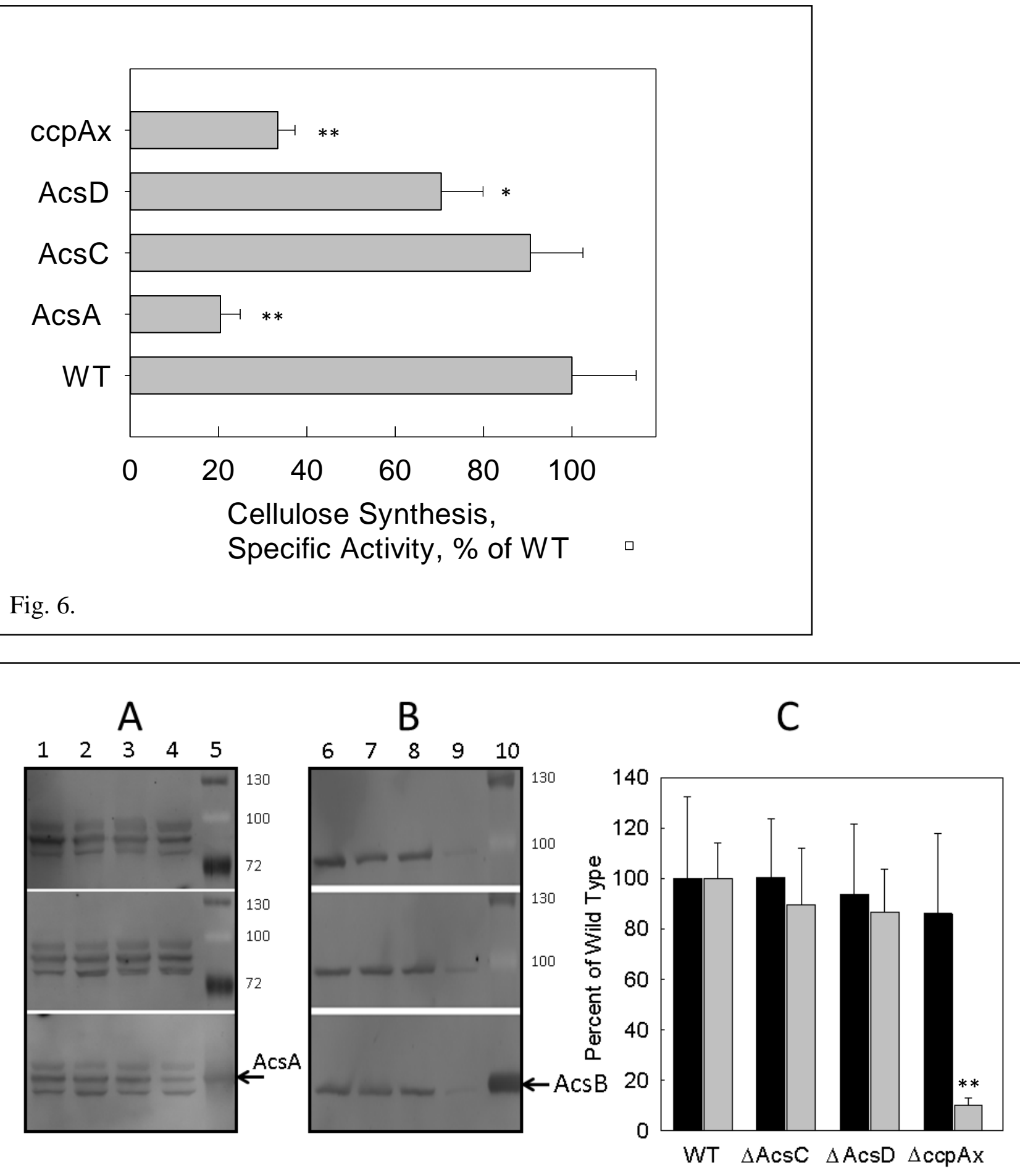

Fig. 7. 


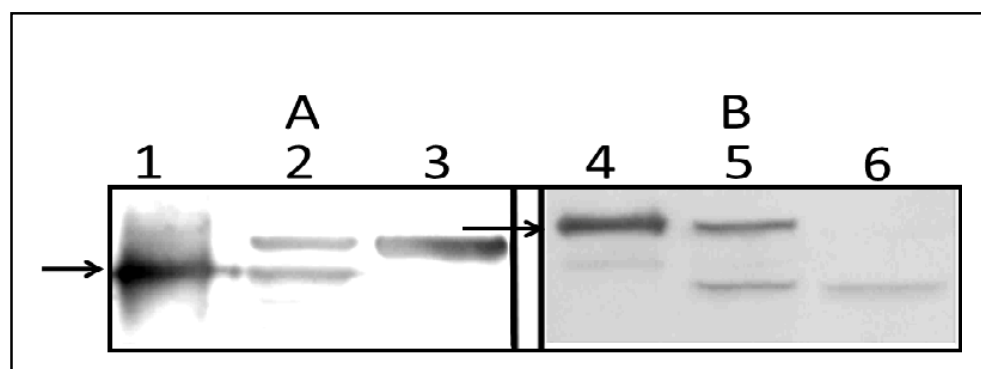

Fig. 8.

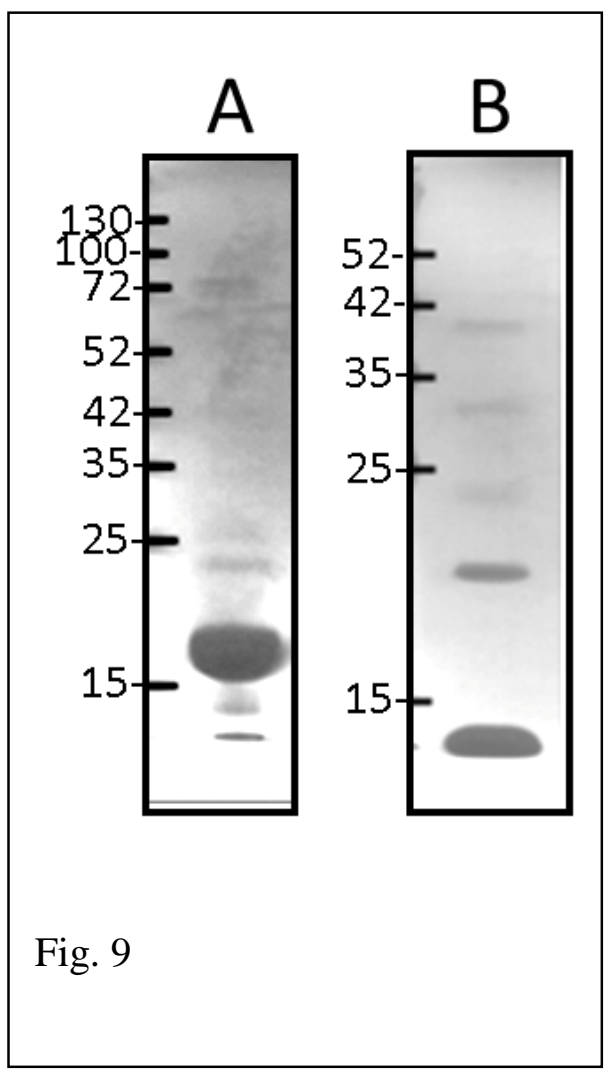

